\title{
Recent advances in the study of uveitis
}

\author{
E. S. PERKINS \\ Institute of Ophthalmology, University of London
}

Contributed by request and dedicated to Sir Stewart Duke-Elder

No attempt will be made here to review the many articles and books on uveitis which have $\overline{\mathrm{G}}$ appeared since Volume IX of the System of Ophthalmology was published in I966. Instead I 응. have chosen a few topics which seem to me to offer the possibility of a greater understanding $\mathcal{O}_{\infty}$ of the underlying pathological mechanisms of intraocular inflammation. Inevitably such a choice is coloured by personal interests, and other compilers would have chosen differently. Readers wishing for a wider review are recommended to consult Schlaegel (1969), Aronson and Elliott (1972), and Campinchi, Fauré, Bloch-Michel, and Haut (r973).

\section{Laboratory studies}

\section{(a) Cellular immunity}

Studies of circulating (humoral) antibodies have been of considerable value in the aetiological diagnosis of uveitis where an infective process such as toxoplasmosis has been primarily involved. The cellular antibody system has been less amenable to study in the clinical situation, but with the development of the lymphocyte transformation test it has become possible to demonstrate cellular hypersensitivity to a variety of antigens. The test is performed by culturing lymphocytes from the blood and observing the process of gradual enlargement, transformation into lymphoblasts, and mitotic activity, which takes place when they come into contact with an antigen to which the organism has previously been sensitized. The rate of stimulation of lymphocytes can be estimated by measuring the increment in $\mathrm{H}^{3}$-thymidine incorporation, and if the ratio of scintillation counts in stimulated and non-stimulated cultures exceeds $I \cdot 5$ the lymphocyte transformation test is considered positive.

Phytohaemagglutinin (PHA) is a non-specific stimulator of lymphocyte transformation 9 but is not effective if the lymphocytes are derived from a subject with impaired immune responses or receiving immunosuppressive treatment.

Hammer (197I) used this technique to compare the effects of uveal pigment and PHA $N$ on lymphocyte cultures from patients with sympathetic ophthalmitis and the Vogt- N Koyanagi-Harada syndrome. Significant stimulation was obtained in the lymphocytes of N two patients with the latter syndrome and three patients with sympathetic ophthalmitis, compared with controls. These results offer support to previous studies, suggesting that uveal pigment acts as an antigen in these conditions and that autoantibody formation to pigment may play a significant role in the pathological process.

Marak, Font, Johnson, and Alepa (I97I), using a similar test, obtained positive results in two of four patients with sympathetic ophthalmitis when lymphocyte cultures were exposed to retinal pigment epithelium; but also obtained positive results with soluble 
antigens from lens and retina in one of the patients. Henley, Okas, Waithe, Hirschhorn, and Leopold (1972) applied the test to patients with chronic virus keratitis and other ocular conditions, including uveitis, but were unable to obtain stimulation with either virus or ocular tissue antigens.

A rather similar test of cellular immunity is the inhibition of leucocyte migration by antigens to which the donor is sensitized, and although Strandgaard and Braendstrup (197I) obtained negative results in 22 patients with uveitis a number of positive results were obtained by Feinberg, Shore, Leopold, and Henley (1972) in patients with uveitis and in some other ocular diseases, using uveal antigen. Henley, Leopold, Okas, and Shore (1973) were also able to demonstrate cellular immunity to choroid and retina in about one-third of patients with diabetic retinopathy.

It must be emphasized that the demonstration of cellular or humoral autoantibodies to uveal or other ocular tissues does not necessarily imply that uveitis is an autoimmune disease. It could be that auto-antibody production is a normal mechanism in the response to tissue damage; only in lens-induced uveitis, and possibly in sympathetic ophthalmitis, is there an indication that autoimmune reactions may play a primary role. However, the increased interest in aspects of cellular immunity may well provide the knowledge required for testing new theories concerning the aetiology of uveitis.

\section{(b) Prostaglandins}

The prostaglandins are a specialized group of hydroxy fatty acids which occur in the natural state in a large number of mammalian tissues. Ambache and his colleagues (Ambache, Kavanagh, and Whiting, 1965) showed that prostaglandin-like substances were released into the aqueous humour of the rabbit eye in response to mechanical trauma; and the application of prostaglandins to the eye results in vasodilatation, increased vascular permeability, and a raised intraocular pressure. The reaction in the eye resembles an acute anterior uveitis, and it has now been shown that, in experimentally induced uveitis in the rabbit, large amounts of a substance with an activity similar to that of prostaglandin $E_{1}$ $\left(\mathrm{PGE}_{1}\right)$ were released into the aqueous with small quantities of a material with an activity like that of $\mathrm{PGE}_{2}$ (Eakins, Whitelocke, Perkins, Bennett, and Unger, 1972).

Examination of aqueous humour from untreated cases of anterior uveitis in man showed significant amounts of prostaglandin-like activity. Little activity was found in aqueous from patients treated with steroids, and none was detected in aqueous from uninflamed eyes of patients with cataract (Eakins, Whitelocke, Bennett, and Martenet, 1972). It is very likely therefore that prostaglandins are responsible for the vasodilatation and increased permeability in eyes with uveitis. It is not clear as yet whether the prostaglandins are derived from uveal tissue or whether they are released from white blood cells which enter the inflamed eye.

These findings are important for two reasons : first, they suggest that the mechanism of anterior uveitis is similar to that of the experimental model in rabbits, in which the inflammation results from an antigen-antibody reaction in the eye; and, secondly, they provide an explanation for the favourable response of the human disease to treatment with indomethacin (Perkins and MacFaul, I965), which is now known to be a potent prostaglandin antagonist (Vane, 197I). The response to steroids may also depend on the inhibition of prostaglandin synthesis (Greaves and McDonald-Gibson, I972). Further work in this field promises to shed new light on the basic mechanisms of ocular inflammation and provide new non-steroid anti-inflammatory drugs. 


\section{Glinical studies}

\section{(a) Ankylosing spondylitis and uveitis}

The association between acute anterior uveitis and rheumatic conditions such as ankylosing $\overrightarrow{\vec{s}}$ spondylitis has been widely recognized for many years, but the reason for this association has remained obscure. The possibility that the link may be genetic is suggested by recent work on tissue-typing antigens. Brewerton, Caffrey, Hart, James, Nicholls, and Sturrock (1973) and Schlosstein, Terasaki, Bluestone, and Pearson (I973) have now shown that a high proportion of patients with ankylosing spondylitis possess one particular blood group antigen HL-A W27. This is a human leucocyte antigen which is only found in some 6 per cent of the normal population but has a higher incidence in the first-degree relatives of patients with ankylosing spondylitis. Uveitis is a common complication of Reiter's disease, and it now appears that a high proportion of patients with this condition also possess HL-A 27 antigen. Zachariae, Hjortshøj, and Kissmeyer-Nielsen (r973) investigated nine male patients with Reiter's disease, five of whom had prostatitis, six conjunctivitis, and one iritis. None had ankylosing spondylitis but two had sacroiliitis. Tissue typing revealed that eight of the nine patients possessed the HL-A 27 antigen. Brewerton, Caffrey, Nicholls, Walters, Oates, and James (I973) studied 33 patients with Reiter's disease and found HL-A 27 in 25, compared with three out of 33 patients with non-specific urethritis, and two out of 33 controls.

Knowing the close association between chronic prostatitis, ankylosing spondylitis, and Reiter's disease with acute anterior uveitis in men (Catterall and Perkins, 196r), it would not be surprising to find that many such patients also have the HL-A 27 antigen. A preliminary study by Brewerton, Caffrey, Nicholls, Walters, and James (1973) has confirmed this expectation by identifying HL-A 27 in 26 out of fifty patients with acute anterior uveitis, compared with two out of fifty controls; 2 I patients had significant associated disease and eighteen of these had HL-A 27. The associated disease was not confined to classical ankylosing spondylitis or Reiter's disease, but included a wide range of previously undiagnosed rheumatic diseases. HL-A 27 was also present in eight out of the 29 patients with no associated disease, five being young women in the 20 to 30 -year age group.

It seems very likely from these results that the possession of HL-A 27 antigen is the common factor in the pathogenesis of ankylosing spondylitis and uveitis. Two possible mechanisms have been suggested by which histocompatibility antigens might influence susceptibility to disease. In one it is postulated that histocompatibility antigens are so similar to the antigens of an invading virus that the host is unable to produce a suitable immune response to the particular virus. In the other. it is believed that specific immune responses, including those to viruses, are inherited, that there may be specific "immune response genes", and that the linkage between histocompatibility antigens and these genes may be a general phenomenon. Much further work will be required before either of these mechanisms can be incriminated in the pathology of uveitis, but the results do provide a fresh stimulus for further research.

\section{(b) Virus studies}

Occasional cases of uveitis have frequently been reported in association with systemic virus infections, but except for the herpes viruses there has so far been little evidence for a virus aetiology in any significant proportion of cases of uveitis. An extensive study by Martenet (197I), in which I 70 cases of uveitis were screened for possible virus infection by examination of serum and aqueous for antibodies, and in a few cases by electron microscopy of biopsy material, revealed a probable virus aetiology in 7 per cent. These comprised 
five cases of herpes simplex, three of herpes zoster, and one of varicella. A virus aetiology was suspected in a further six cases (rickettsia I, adenovirus 3, infectious mononucleosis 2). Although these results are interesting they do not suggest that viruses play a major role in the aetiology of uveitis, but it must be remembered that only a limited range of viruses has been studied as yet. Possibly a search for chlamydial infection would be more rewarding, as there is evidence that some cases of Reiter's disease may be caused by these organisms (Dunlop, in press)

\section{(c) Syphilis}

Considerable interest was aroused by reports of the presence of Treponema pallidum-like spirochaetes in tissues and body fluids (including aqueous) in patients with a history of late, treated syphilis and in the aqueous humour of patients with apparently non-specific intraocular inflammation (for references see Ryan, Nell, and Hardy, 1972). The pathogenicity of the observed organism has, however, never been proved and a careful study by Ryan and others (1972) casts considerable doubt on the significance of previous observations. They studied the aqueous humour from 153 patients but were unable to detect viable organisms in any specimen. Remains of spirochaetal organisms were seen in only three patients and in only one of these were they morphologically similar to $T$. pallidum. The authors conclude that small spiral objects in aqueous humour are unrelated to syphilis.

The earlier findings suggesting a syphilitic origin for uveitis in patients with no other clinical evidence and negative serology must be re-evaluated in the light of this new evidence.

\section{(d) Uveitis in association with multiple sclerosis}

Reports on the association of retinal periphlebitis with multiple sclerosis have been conflicting since the original finding of sheathing of retinal veins by Rucker (1944). Cases of posterior uveitis accompanying the retinal vessel sheathing (Archambeau, Hollenhorst, and Rucker, 1965) and peripheral uveitis-pars planitis-in which sheathing of retinal veins is common, appear also to be associated with multiple sclerosis (Breger and Leopold, ı966; Giles, 1970).

A review of sixty patients with multiple sclerosis (Porter, 1972) substantiates the view that uveitis occurs with increased frequency among such cases. He found nine with evidence of uveitis, in seven of which there was sheathing of the retinal veins. One other had a fibrous plaque overlying a retinal vein, and another had an old inactive patch of choroiditis at the periphery. Five of the cases with uveitis had some evidence of an anterior uveitis with flare and cells in the aqueous and posterior synechiae.

The coexistence of two relatively uncommon conditions is suggestive of a common aetiology; in both diseases autoimmune mechanisms or virus infections have been suggested as causal factors. It may be that uveitis and demyelinating disease are genetically determined reactions of susceptible persons to an as yet undetected virus infection.

\section{Treatment}

\section{Immunosuppressive therapy in uveitis}

Drugs such as methotrexate, azathioprine, cyclophosphamide, and chlorambucil have been used sporadically in the treatment of cases of severe uveitis for the last ro years, and the reports to 1969 have been well reviewed by Campinchi and others (1973). From these uncontrolled clinical trials it seems that a proportion of patients have improved and that the best results have been in cases of sympathetic ophthalmitis and Behçet's disease. As yet 
however there is no consensus of opinion as to which drug is best and what are the indications for immunosuppressive treatment. Only one double-blind controlled trial of azathioprine in chronic iridocyclitis has so far been published (Mathews, Crawford, Bignell, and Mackay, I969), and the results of this careful study on nineteen patients are a salutary lesson in the need for controlled trials in a condition which varies spontaneously in activity. One out of eight control patients and five of eleven treated patients claimed symptomatic improvement, but objective assessment of visual acuity, uveal activity, and intraocular pressure failed to show any significant differences between controls and treated cases.

It should be emphasized that the number of cases in this trial is small and that some benefit of azathioprine might have emerged if the series had been larger. Some of the results from uncontrolled trials do seem to suggest quite dramatic improvement in individual cases of uveitis, particularly in cases of sympathetic ophthalmitis (Lazar, Weiner, and Leopold, ı969; Verdaguer and Valenzuela, I968; Moore, I968) and Behçet's disease (Rosselet, Saudan, and Zenklusen, I968; Abdalla and Bahgat, I973). Further experience may give a clearer idea of the conditions most likely to benefit from immunosuppressive therapy. The possible benefits must however be weighed against the toxic side-effects of these drugs and treatment should be reserved for progressive cases which fail to respond to adequate steroid therapy.

Although the studies described above do not offer an immediate solution to the clinical $\vec{\varphi}$ management of uveitis, the developments in immunopathology, the association of uveitis with genetically determined tissue antigens, and the demonstration of prostaglandins in the aqueous suggest at least an advance in our understanding of this complex disease.

\section{References}

ABDalla, M. I., and Bahgat, N. EL-D. (1973) Brit. J. Ophthal., 57, 706

ambache, N., kavanagh, L., and whiting, J. (1965) $\mathcal{F}$. Physiol. (Lond.), i 76, 378

ARGhambeau, P. L., hollenhorst, R. W., and RuGker, c. W. (ig65) Proc. Mayo Clin., 40, 544

ARONSON, S. B., and ELliotT, J. H. (1972) "Ocular Inflammation". Kimpton, London

BREger, B. C., and LeOPOLD, I. H. (I966) Amer. 7. Ophthal., 62, 540

BREWERTON, D. A., GAFFrEY, M., HART, F. DUDLEY, JAMES, D. C. O., NICHOLLS, A., and STURROCK, R. D.

(1973) Lancet, I, 904

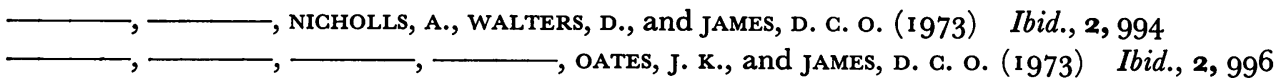

CAMPINCHI, R., FAURÉ, J. P., BLOCH-MICHEL, E., and HAUT, J. (I973) “Uveitis: Immunologic and I

Allergic Phenomena". Thomas, Springfield, Ill.

CATTERAll, R. D., and Perkins, E. s. (196r) Brit. F. Ophthal., 45, 109

DUNLOP, E. M. G. "Recent Advances in Venereology" (in press)

EAKins, K. E., Whitelocke, R. A. F., BenNetT, A., and MARTENet, A. c. (i972) Brit. med. $7 ., 3,452$

248

FEINBERG, E. B., SHORE, B., LEOPOLD, I. H., and HENLEy, W. L. (I972) Amer. 7. Ophthal., 73, 68

GILES, C. L. (I970) Ibid., 70, I 7

GREAVES, M. W., and MCDonald-Gibson, w. (I972) Brit. med. F., 2, 83

HAMMER, H. (I97I) Brit. 7. Ophthal., 55, 850

HENLEy, W. L., LEOPOld, I. H., OKAS, s., and SHORE, B. (I973) Amer. F. Ophthal., 76, 279

- OKAS, s., WAITHE, W., HIRSCHHORN, K., and LEOPOLD, I. H. (I972) Ibid., 73, 56

LAZAR, M., WEINER, M. J., and LEOPOLD, I. H. (I969) Ibid., 67, 383

MARAK, G. E., FONT, R. L., JOHNSON, M. C., and ALEPA, F. P. (197I) Invest. Ophthal., ro, 770 
MARTEnet, A. C. (197I) "Virus et uvéites". Doin, Paris MATHEWS, J. D., CRAWFORD, B. A., BIGNELl, J.ฯ., and MAGKAy, I. R. (I969) Brit. J. Ophthal., 53, 327 MOORE, C. E. (I968) Ibid., 52, 688

PERKins, E. S., and Macfaul, P. A. (1965) Trans. ophthal. Soc. U.K., 85, 53 PORTER, R. (I972) Brit. F. Ophthal., 56, 478

ROSSELET, E., SAUDAN, Y., and zeNKLUSEN, G. (1968) Ophthalmologica (Basel), I56, 2 I 8

RUGKer, c. W. (1944) Proc. Mayo Clin., r9, i 76

RYAN, s. J., NEll, E. E., and hardy, P. H. (1972) Amer. F. Ophthal., 73, 250

SCHLAEGEL, T. F. (I969) 'Essentials of Uveitis". Churchill, London

Schlosstein, L., terasaki, P. I., Bluestone, R., and pearson, c. M. (I973) New Engl. 7. Med., 288, 704

STRANDGAARD, s., and BRAENDSTRUP, O. (I97I) Acta ophthal. (Kbh.), 49, 768

VANE, J. R. (197I) Nature (New Biol.), 23I, 232

verdaguer, J., and valenzuela, H. (1968) Arch. chil. Oftal., 25, 80

ZAGHARIAE, H., HJORTSHøJ, A., and KISSMEYER-NIELSEN, F. (I973) Lancet, 2, 565 